\title{
Implicit numerical scheme based on SMAC method for unsteady incompressible Navier-Stokes equations
}

\author{
Li Zhenlin* and Zhang Yongxue \\ School of Mechanical and Electronic Engineering, China University of Petroleum, Beijing 102249, China
}

\begin{abstract}
An implicit numerical scheme is developed based on the simplified marker and cell (SMAC) method to solve Reynolds-averaged equations in general curvilinear coordinates for three-dimensional (3-D) unsteady incompressible turbulent flow. The governing equations include the Reynolds-averaged momentum equations, in which contravariant velocities are unknown variables, pressure-correction Poisson equation and $k-\varepsilon$ turbulent equations. The governing equations are discretized in a 3-D MAC staggered grid system. To improve the numerical stability of the implicit SMAC scheme, the higherorder high-resolution Chakravarthy-Osher total variation diminishing (TVD) scheme is used to discretize the convective terms in momentum equations and $k-\varepsilon$ equations. The discretized algebraic momentum equations and $k-\varepsilon$ equations are solved by the time-diversion multiple access (CTDMA) method. The algebraic Poisson equations are solved by the Tschebyscheff SLOR (successive linear over relaxation) method with alternating computational directions. At the end of the paper, the unsteady flow at high Reynolds numbers through a simplified cascade made up of NACA65-410 blade are simulated with the program written according to the implicit numerical scheme. The reliability and accuracy of the implicit numerical scheme are verified through the satisfactory agreement between the numerical results of the surface pressure coefficient and experimental data. The numerical results indicate that Reynolds number and angle of attack are two primary factors affecting the characteristics of unsteady flow.
\end{abstract}

Key words: Unsteady incompressible flow, SMAC method, contravariant velocity, TVD scheme, cascade

\section{Introduction}

Much interest in computational fluid dynamics is focused on the simulation of practical flow in complex geometry. Unsteady flow phenomena, flow separation, recirculation, vortex shedding, turbulence, etc. often occur in practical flow. It is a formidable task to simulate the practical flow accurately. Ghia et al (1989) developed an unsteady direct solution method in general curvilinear orthogonal coordinates by using the vorticity and stream function. Rao et al (1992) presented a primitive variables solution method by using Cartesian velocity components. In this method, the third-order upwind scheme was adopted to discretize the convective terms. Rosenfeld et al (1991) developed a fractional step solution method to determine 3-D viscous flow in a generalized coordinates system. Rogers (1995) extended the artificial compressibility methods to unsteady incompressible flow by introducing the second-order backward-difference scheme and the Newton iteration with dual-time stepping to restore the time accuracy of the solution. For these methods velocity and pressure are taken as unknown variables, which is advantageous to numerical simulation of turbulent flow.
The conventional turbulence models can be used directly. The MAC (marker and cell) method (Harlow and Welch $1965)$ is one of the primitive variables methods to solve incompressible flow. The SMAC method (Amsden and Harlow, 1970) was developed based on the MAC method. It inherits the two characteristics of the MAC method, including using an MAC staggered grid and computing Poisson equation. However, the SMAC method is more stable than the MAC method and is advanced in computing Poisson equation (Zhang et al, 2004). In this paper, an implicit SMAC scheme in general curvilinear coordinates for unsteady incompressible flow was developed. To validate the scheme, the unsteady flow of a 2-D cascade was calculated. The structures of flow fields and the mechanism of unsteady flow were investigated preliminarily.

\section{Governing equations}

The fundamental equations for unsteady incompressible turbulent flow are Reynolds-averaged N-S equations and continuity equation, which are functions of contravariant velocity, in general curvilinear coordinates. They can be written in conservative form (Zhang, 2005a):

\footnotetext{
* Corresponding author. E-mail: zhenlinli@263.net

Received December 20, 2007
} 


$$
\begin{gathered}
\partial\left(J U_{l}\right)+\frac{\partial\left(J U_{i} U_{l}\right)}{\partial \xi_{i}}-\boldsymbol{u} \cdot\left[J U_{i} \frac{\partial}{\partial \xi_{i}}\left(\nabla \xi_{l}\right)\right]=F_{l}-J g_{l i} \frac{\partial p}{\partial \xi_{i}}-v \varepsilon_{l i j} \frac{\partial\left(h_{j k} Z_{k}\right)}{\partial \xi_{i}}+J \alpha_{i j k l} \frac{\partial\left(\overline{u_{i}^{\prime} u_{j}^{\prime}}\right)}{\partial \xi_{k}} \quad(l=1,2,3) \\
\frac{\partial}{\partial \xi_{i}}\left(J U_{i}\right)=0
\end{gathered}
$$

where $t$ is time. $\quad \xi_{i}$ is general curvilinear coordinate component; $U_{i}$ is contravariant velocity component, $U_{i}=\nabla \xi_{i} \cdot \boldsymbol{u}$; $u$ is physical velocity vector; $p$ is pressure, $v$ is kinetic viscosity; $\varepsilon_{i j k l}$ is the Levy-Civita permutation tensor; the metrics $g_{i j}$ and $h_{i j}$ are contravariant tensor components and covariant tensor components, $g_{i j}=\nabla \xi_{i} \cdot \nabla \xi_{j}, h_{i j}=\frac{\partial x_{k}}{\partial \xi_{i}} \frac{\partial x_{k}}{\partial \xi_{j}} ; \alpha_{i j k l}$ is defined as $\alpha_{i j k l}=\frac{\partial \xi_{l}}{\partial x_{i}} \frac{\partial \xi_{k}}{\partial x_{j}} ; J$ is Jacobian, $J=\frac{\partial(x, y, z)}{\partial(\xi, \eta, \zeta)} ; F_{l}$ is inertial force; $Z_{k}$ is contravariant vorticity, $Z_{k}=\nabla \xi_{i} \cdot \Omega, \Omega$ is vorticity vector, $\Omega=\nabla \times \boldsymbol{u}$.

It is necessary to introduce the turbulence model to close Eqs. (1) and (2). In this paper, the standard $k$ - $\varepsilon$ turbulence model (Hou and Zou, 2005) was adopted. The $k-\varepsilon$ equations in general curvilinear coordinates can be written as follows:

$$
\begin{gathered}
\frac{\partial}{\partial t}(J k)+\frac{\partial}{\partial \xi_{i}}\left(J U_{i} k\right)=\frac{\partial}{\partial \xi_{i}}\left[\left(v+\frac{v_{T}}{\sigma_{k}}\right) J g_{i j} \frac{\partial k}{\partial \xi_{j}}\right]+J(P-\varepsilon) \\
\frac{\partial}{\partial t}(J \varepsilon)+\frac{\partial}{\partial \xi_{i}}\left(J U_{i} \varepsilon\right)=\frac{\partial}{\partial \xi_{i}}\left[\left(v+\frac{v_{T}}{\sigma_{\varepsilon}}\right) J g_{i j} \frac{\partial \varepsilon}{\partial \xi_{j}}\right]+J\left(C_{\varepsilon 1} \frac{\varepsilon}{k} P-C_{\varepsilon 2} \frac{\varepsilon^{2}}{k}\right)
\end{gathered}
$$

where $k, \varepsilon, P$ and $v_{T}$ are the turbulence kinetic energy, the dissipation rate of $k$, the production of $k$ and the eddy viscosity, respectively. They are defined as: $k=\frac{1}{2} \overline{u_{i}^{\prime} u_{i}^{\prime}}, \varepsilon=\frac{\partial u_{i}^{\prime}}{\partial x_{l}} \frac{\partial u_{i}^{\prime}}{\partial x_{l}}, v_{T}=C_{\mu} \frac{k^{2}}{\varepsilon}, P=v_{T}\left(\frac{\partial u_{i}}{\partial x_{j}}+\frac{\partial u_{j}}{\partial x_{i}}\right) \frac{\partial u_{i}}{\partial x_{j}}$. The coefficients $C_{\mu}=0.09, \sigma_{k}=1.0, C_{\varepsilon 1}=1.44, C_{\varepsilon 2}=1.92$ and $\sigma_{\varepsilon}=1.3$.

The usual SMAC scheme for incompressible N-S equations in general curvilinear coordinates is explicit. It can be used directly and easily for calculation. However, in the explicit scheme, the time increment $\Delta t$ is strictly limited to satisfy the CFL number. To improve the numerical stability and computational efficiency, the implicit scheme developed for solving steady flow (Zhang et al, 2005b) was extended to unsteady flow (Zhang et al, 2006). The governing equations (including momentum equations, $k$ equation, $\varepsilon$ equation and Poisson equation) of implicit SMAC scheme for three-dimensional unsteady incompressible turbulent flow can be written as follows:

$$
\begin{aligned}
& \left\{1+\frac{\Delta t}{2}\left[J U^{(m)} \frac{\partial}{\partial \xi} \frac{1}{J}+v \frac{\partial}{\partial \xi}\left(\frac{h_{23}}{J} \frac{\partial}{\partial \xi} \frac{h_{23}}{J}-\frac{h_{33}}{J} \frac{\partial}{\partial \xi} \frac{h_{22}}{J}\right)\right]\right\} \cdot\left\{1+\frac{\Delta t}{2}\left[J V^{(m)} \frac{\partial}{\partial \eta} \frac{1}{J}+v \frac{\partial}{\partial \eta}\left(\frac{h_{13}}{J} \frac{\partial}{\partial \eta} \frac{h_{13}}{J}-\frac{h_{33}}{J} \frac{\partial}{\partial \eta} \frac{h_{11}}{J}\right)\right]\right\} \cdot \\
& \left\{1+\frac{\Delta t}{2}\left[J W^{(m)} \frac{\partial}{\partial \zeta} \frac{1}{J}+v \frac{\partial}{\partial \zeta}\left(\frac{h_{12}}{J} \frac{\partial}{\partial \zeta} \frac{h_{12}}{J}-\frac{h_{22}}{J} \frac{\partial}{\partial \zeta} \frac{h_{11}}{J}\right)\right]\right\} \Delta J U^{*(m)}=R H S_{1}^{*(m-1)} \\
& \left\{1+\frac{\Delta t}{2}\left[J U^{(m)} \frac{\partial}{\partial \xi} \frac{1}{J}+v \frac{\partial}{\partial \xi}\left(\frac{h_{23}}{J} \frac{\partial}{\partial \xi} \frac{h_{23}}{J}-\frac{h_{33}}{J} \frac{\partial}{\partial \xi} \frac{h_{22}}{J}\right)\right]\right\} \cdot\left\{1+\frac{\Delta t}{2}\left[J V^{(m)} \frac{\partial}{\partial \eta} \frac{1}{J}+v \frac{\partial}{\partial \eta}\left(\frac{h_{13}}{J} \frac{\partial}{\partial \eta} \frac{h_{13}}{J}-\frac{h_{11}}{J} \frac{\partial}{\partial \eta} \frac{h_{33}}{J}\right)\right]\right\} \cdot \\
& \left\{1+\frac{\Delta t}{2}\left[J W^{(m)} \frac{\partial}{\partial \zeta} \frac{1}{J}+v \frac{\partial}{\partial \zeta}\left(\frac{h_{12}}{J} \frac{\partial}{\partial \zeta} \frac{h_{12}}{J}-\frac{h_{11}}{J} \frac{\partial}{\partial \zeta} \frac{h_{22}}{J}\right)\right]\right\} \Delta V^{*(m)}=R H S_{2}^{*(m-1)} \\
& \left\{1+\frac{\Delta t}{2}\left[J U^{(m)} \frac{\partial}{\partial \xi} \frac{1}{J}+v \frac{\partial}{\partial \xi}\left(\frac{h_{23}}{J} \frac{\partial}{\partial \xi} \frac{h_{23}}{J}-\frac{h_{22}}{J} \frac{\partial}{\partial \xi} \frac{h_{33}}{J}\right)\right]\right\} \cdot\left\{1+\frac{\Delta t}{2}\left[J V^{(m)} \frac{\partial}{\partial \eta} \frac{1}{J}+v \frac{\partial}{\partial \eta}\left(\frac{h_{13}}{J} \frac{\partial}{\partial \eta} \frac{h_{13}}{J}-\frac{h_{11}}{J} \frac{\partial}{\partial \eta} \frac{h_{33}}{J}\right)\right]\right\} . \\
& \left\{1+\frac{\Delta t}{2}\left[J W^{(m)} \frac{\partial}{\partial \zeta} \frac{1}{J}+v \frac{\partial}{\partial \zeta}\left(\frac{h_{12}}{J} \frac{\partial}{\partial \zeta} \frac{h_{12}}{J}-\frac{h_{22}}{J} \frac{\partial}{\partial \zeta} \frac{h_{11}}{J}\right)\right]\right\} \Delta J W^{*(m)}=R H S_{3}^{*(m-1)}
\end{aligned}
$$




$$
\begin{gathered}
\left(1+\frac{\Delta t}{2} J U^{(m)} \frac{\partial}{\partial \xi} \frac{1}{J}\right) \cdot\left(1+\frac{\Delta t}{2} J V^{(m)} \frac{\partial}{\partial \eta} \frac{1}{J}\right) \cdot\left(1+\frac{\Delta t}{2} J W^{(m)} \frac{\partial}{\partial \zeta} \frac{1}{J}\right) \Delta J k^{(m)}=R H S_{k}^{*(m-1)} \\
\left(1+\frac{\Delta t}{2} J U^{(m)} \frac{\partial}{\partial \xi} \frac{1}{J}\right) \cdot\left(1+\frac{\Delta t}{2} J V^{(m)} \frac{\partial}{\partial \eta} \frac{1}{J}\right) \cdot\left(1+\frac{\Delta t}{2} J W^{(m)} \frac{\partial}{\partial \zeta} \frac{1}{J}\right) \Delta J \varepsilon^{(m)}=R H S_{\varepsilon}^{*(m-1)} \\
\frac{\partial}{\partial \xi_{l}}\left(J g_{l i} \frac{\partial \phi^{(m)}}{\partial \xi_{i}}\right)=\frac{2}{\Delta t} \frac{\partial}{\partial \xi_{l}}\left(J U_{l}^{*(m)}\right)
\end{gathered}
$$

where if $l=1,2,3, \quad R H S_{l}^{*(0)}=-\Delta t\left(C_{l}+D_{l}+P_{l}+A_{l}+R_{l}\right)^{n}, \operatorname{RHS}_{l}^{*(m)}=R H S_{l}^{*(m-1)}+\Delta R H S_{l}^{*(m)} \quad(m \geq 1)$

$$
\begin{gathered}
\Delta R H S_{l}^{*(m)}=-\left(J U_{l}^{(m)}-J U_{l}^{(m-1)}\right)-\frac{1}{2} \Delta t\left(F_{l}^{(m)}-F_{l}^{(m-1)}\right), F_{l}=C_{l}+D_{l}+P_{l}+R_{l} \\
C_{l}=\frac{\partial\left(J U_{i} U_{l}\right)}{\partial \xi_{i}}, A_{l}=-\boldsymbol{u} \cdot\left[J U_{i} \frac{\partial\left(\nabla \xi_{l}\right)}{\partial \xi_{i}}\right], D_{l}=v \varepsilon_{l i j} \frac{\partial\left(h_{j k} Z_{k}\right)}{\partial \xi_{i}}, P_{l}=J g_{l i} \frac{\partial p}{\partial \xi_{i}}, R_{l}=-J \alpha_{i j k l} \frac{\partial}{\partial \xi_{k}}\left[v_{T}\left(\frac{\partial u_{i}}{\partial x_{j}}+\frac{\partial u_{j}}{\partial x_{i}}\right)\right]
\end{gathered}
$$

If $l=k, \varepsilon, R H S_{l}^{*(0)}=-\Delta t\left(C_{l}+D_{l}+G_{l}\right)^{n}, R H S_{l}^{*(m)}=R H S_{l}^{*(m-1)}+\Delta R H S_{l}^{*(m)} \quad(m \geq 1)$

$$
\begin{aligned}
& \Delta R H S_{l}^{*(m)}=-\left(J l^{(m)}-J l^{(m-1)}\right)-\frac{1}{2} \Delta t\left(F_{l}^{(m)}-F_{l}^{(m-1)}\right), \quad F_{l}=C_{l}+D_{l}+G_{l} \\
& C_{l}=\frac{\partial}{\partial \xi_{i}} J U_{i} l, \quad D_{l}=-\frac{\partial}{\partial \xi_{i}}\left[\left(v+\frac{v_{T}}{\sigma_{l}}\right) J g_{i j} \frac{\partial l}{\partial \xi_{j}}\right], \quad G_{k}=-J(P-\varepsilon), G_{\varepsilon}=-J\left(C_{\varepsilon 1} \frac{\varepsilon}{k} P-C_{\varepsilon} \frac{\varepsilon^{2}}{k}\right)
\end{aligned}
$$

The primary difference of implicit SMAC scheme for unsteady and steady incompressible turbulent flow is the $m$ times Newton iterations between $n$ and $n+1$ time step for variables. The superscript $(m)$ denotes the $m$-th approximation of $n+1$ time step variables. The computational procedure at each time step for the advancing solution with a time increment $\Delta \mathrm{t}$ is as follows.

I. Compute $\Delta J l^{*(m)}(l=1,2,3, k, \varepsilon)$ from Eqs. (5)- (9).

II. Compute $J U_{l}^{*(m)}(l=1,2,3), \quad J U_{l}^{*(m)}=J U_{l}^{(m-1)}+\Delta J U_{l}^{*(m)}$.

III. Compute $\phi^{(m)}$ from Eq. (10).

IV. Compute $J U_{l}^{(m)}=J U_{l}^{*(m)}-\frac{1}{2} \Delta t J g_{l i} \frac{\partial \phi^{(m)}}{\partial \xi_{i}}(l=1,2,3)$,

$J l^{(m)}=J l^{(m-1)}+\Delta J l^{(m)}(l=k, \varepsilon), \quad p^{(m)}=p^{(m-1)}+\phi^{(m)}$.

The procedure is repeated at each time step until the convergent result is obtained.

\section{Discretization and algorithm}

The governing equations of implicit SMAC scheme for unsteady incompressible flow are discretized in the MAC staggered grid as shown in Fig. 1.

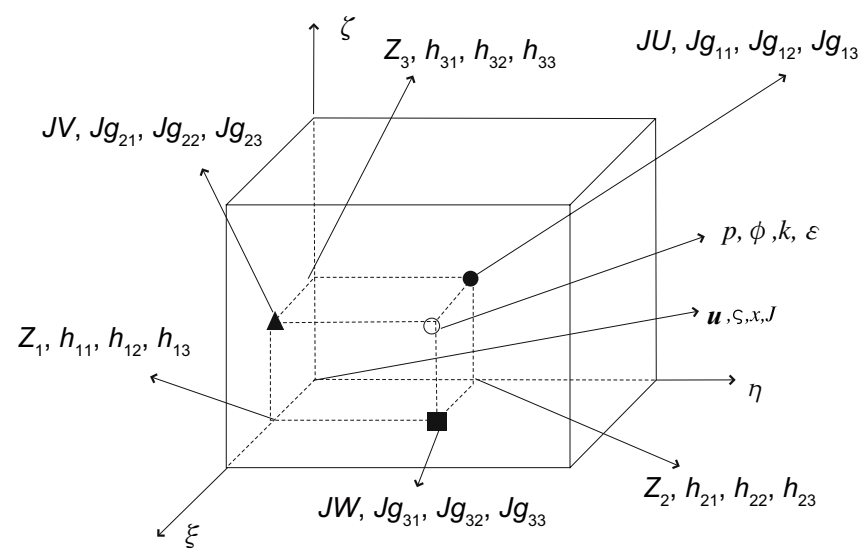

Fig. 1 Three-dimensional staggered grid and definition points of variables

The momentum equations (5), (6), (7), $k$ equation (8) and $\varepsilon$ equation (9) can be solved in the similar process by dividing into three steps, and applying the first-order upstream-difference to the convective terms in the left sides. The accuracy of $\Delta J l^{*(m)}(l=U, V, W, k, \varepsilon)$ depends mainly on the numerical approximation $R H S_{l}^{*(m-1)}$, and $R H S_{l}^{*(m-1)}$ is affected mostly by the convective term $R H S_{I C}{ }^{*(m-1)}$. The higherorder high-resolution Chakravarthy-Osher TVD scheme is introduced to discretize $R H S_{I C}{ }^{*(m-1)}$ (Yonamine et al, 2006). For example, $l=U$, in the general curvilinear coordinates, $\Delta \xi=\Delta \eta=\Delta \zeta=h$, then 


$$
\left(R H S C_{1 C}^{*(m-1)}\right)_{i, j, k U}=\frac{\left(J U^{2}\right)_{i, j, k P}^{(m-1)}-\left(J U^{2}\right)_{i-1, j, k P}^{(m-1)}}{h}+\frac{(J U V)_{i, j+1, k Z_{3}}^{(m-1)}-(J U V)_{i, j, k Z_{3}}^{(m-1)}}{h}+\frac{(J U W)_{i, j, k+1 Z_{2}}^{(m-1)}-(J U W)_{i, j, k Z_{2}}^{(m-1)}}{h}
$$

with

$$
\begin{aligned}
& \left(J U^{2}\right)_{i, j, k P}^{(m-1)}=\frac{1}{2} U_{i, j, k P}^{(m-1)}\left(J U_{i, j, k}^{(m-1)}+J U_{i+1, j, k}^{(m-1)}\right)-\frac{1}{2}\left(D f_{i+1 / 2, j, k}^{+}-D f_{i+1 / 2, j, k}^{-}\right)- \\
& \frac{1-\phi}{4} \min \bmod \left[D f_{i+3 / 2, j, k}^{-}, b D f_{i+1 / 2, j, k}^{-}\right]-\frac{1+\phi}{4} \min \bmod \left[D f_{i+1 / 2, j, k}^{-}, b D f_{i+3 / 2, j, k}^{-}\right]+ \\
& \frac{1+\phi}{4} \min \bmod \left[D f_{i+1 / 2, j, k}^{+}, b D f_{i-1 / 2, j, k}^{+}\right]+\frac{1-\phi}{4} \min \bmod \left[D f_{i-1 / 2, j, k}^{+}, b D f_{i+1 / 2, j, k}^{+}\right] \\
& (J U V)_{i, j, k Z_{3}}^{(m-1)}=\frac{1}{2} V_{i, j, k Z_{3}}^{(m-1)}\left(J U_{i, j-1, k}^{(m-1)}+J U_{i, j, k}^{(m-1)}\right)-\frac{1}{2}\left(D f_{i, j-1 / 2, k}^{+}-D f_{i, j-1 / 2, k}^{-}\right)- \\
& \frac{1-\phi}{4} \min \bmod \left[D f_{i, j+1 / 2, k}^{-}, b D f_{i, j-1 / 2, k}^{-}\right]-\frac{1+\phi}{4} \min \bmod \left[D f_{i, j-1 / 2, k}^{-}, b D f_{i, j+1 / 2, k}^{-}\right]+ \\
& \frac{1+\phi}{4} \min \bmod \left[D f_{i, j-1 / 2, k}^{+}, b D f_{i, j-3 / 2, k}^{+}\right]+\frac{1-\phi}{4} \min \bmod \left[D f_{i, j-3 / 2, k}^{+}, b D f_{i, j-1 / 2, k}^{+}\right] \\
& (J U W)_{i, j, k Z_{2}}^{(m-1)}=\frac{1}{2} W_{i, j, k Z_{2}}^{(m-1)}\left(J U_{i, j, k}^{(m-1)}+J U_{i, j, k-1}^{(m-1)}\right)-\frac{1}{2}\left(D f_{i, j, k-1 / 2}^{+}-D f_{i, j, k-1 / 2}^{-}\right)- \\
& \frac{1-\phi}{4} \min \bmod \left[D f_{i, j, k+1 / 2}^{-}, b D f_{i, j, k-1 / 2}^{-}\right]-\frac{1+\phi}{4} \min \bmod \left[D f_{i, j, k-1 / 2}^{-}, b D f_{i, j, k+1 / 2}^{-}\right]+ \\
& \frac{1+\phi}{4} \min \bmod \left[D f_{i, j, k-1 / 2}^{+}, b D f_{i, j, k-3 / 2}^{+}\right]+\frac{1-\phi}{4} \min \bmod \left[D f_{i, j, k-3 / 2}^{+}, b D f_{i, j, k-1 / 2}^{+}\right] \\
& D f_{i+1 / 2, j, k}^{ \pm}=\left(U_{i, j, k P}^{(m-1)} \pm\left|U_{i, j, k P}^{(m-1)}\right|\right)\left(J U_{i+1, j, k}^{(m-1)}-J U_{i, j, k}^{(m-1)}\right) / 2 \\
& D f_{i, j-1 / 2, k}^{ \pm}=\left(V_{i, j, k Z_{3}}^{(m-1)} \pm\left|V_{i, j, k Z_{3}}^{(m-1)}\right|\right)\left(J U_{i, j, k}^{(m-1)}-J U_{i, j-1, k}^{(m-1)}\right) / 2 \\
& D f_{i, j, k-1 / 2}^{ \pm}=\left(W_{i, j, k Z_{2}}^{(m-1)} \pm\left|W_{i, j, k Z_{2}}^{(m-1)}\right|\right)\left(J U_{i, j, k}^{(m-1)}-J U_{i, j, k-1}^{(m-1)}\right) / 2
\end{aligned}
$$

The flux limiter function is defined as min mod. $(x, y)=\operatorname{sign}(x) \max [0, \min (|x|, y \operatorname{sign}(x))]$. The subscripts $U, V, W, P$ and $\mathrm{X}$ present the points where $J U, J V, J W, p$ and $x$ are defined respectively. The Chakravarthy-Osher TVD scheme is third-order upwind difference while $\phi=1 / 3$ and $b=4$.

The discretized linear equations of Eqs. (5)-(9) at each step have a diagonally dominant tridiagonal matrix, and can be computed easily by the time-diversion multiple access (TDMA) method (Li et al, 2004). The Poisson equation (Eq.(10)) is discretized by second-order central-difference scheme. The corresponding linear equations are computed by SLOR (Successive linear over relaxation) method (Woznicki and Jedrzejec, 2001), and accelerated by ADI method (Ismail and Allan, 2004).

\section{Application to unsteady cascade flow}

A numerical simulation of unsteady 2-D cascade flow was performed using the present implicit SMAC scheme.
The blade section in cascade with a pitch-chord ratio 1.0 was NACA 65-410 (Emery et al, 1958). The settled angle of cascade was $30^{\circ}$. The $\mathrm{H}$-shaped grid was generated algebraically as shown in Fig. 2, where the grid was concentrated near the blade surface and the trail of the blade in order to simulate the boundary layer and the wake flow accurately.

The periodic conditions were imposed on the cyclic boundaries in the upstream and downstream regions. The Neuman condition for the pressure and the no-slip condition were also given on the blade boundaries. The uniform velocity profile was given on the inlet boundary and $p=0$ on the outlet boundary. The $k$ and $\varepsilon$ were given directly by the velocity profile on the inlet boundary. On the blade boundary, the $k$ and $\varepsilon$ were determined by wall function. All of the other boundaries were given Neuman condition of unknown variables.

The computation was carried out with non-dimensional equations. Reynolds number is defined as $R e=u C / v, u$ is the uniform velocity magnitude on the inlet boundary; $C$ is chord 


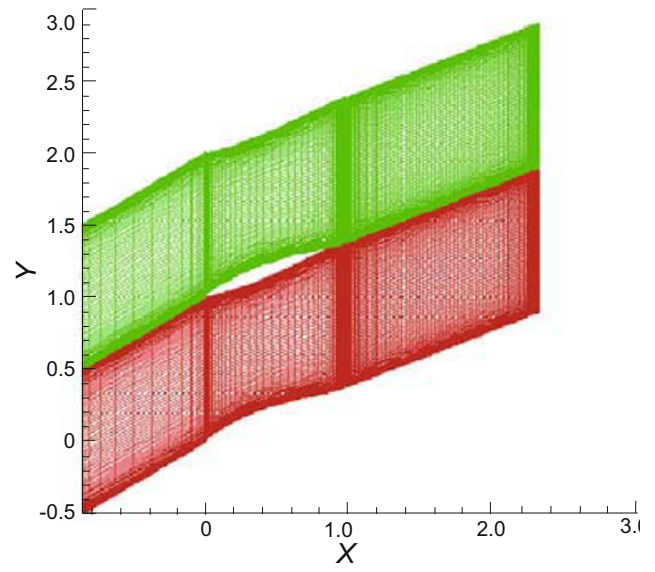

Fig. 2 Computational grid $(151 \times 75)$ length. The numerical results for the angle of attack $\alpha=9^{\circ}$ and $R e=10^{3}$ by using $121 \times 55$ grid points are shown in Fig. 3 . With this Reynolds number, the boundary layer flow and wake flow are simulated well. There are no separation both on suction side and pressure side of the blade.

The velocity vector, pressure contours and the surface pressure distribution of the cascade flow for the angle of attack $\alpha=9^{\circ}$ and $R e=10^{3}$ by using $151 \times 75$ grid points are shown in Fig. 4. The flow becomes unsteady as indicated in Fig. 4. The detailed flow aspect near the trailing edge is shown in this figure. The Kármán Vortex Street is formed in the downstream of cascade. The separation point is at the suction side of the blade, which is two-third chord length to the head of the blade. In Fig. 5, the unsteady cascade flow is shown at different times. At the dimensionless time $t=0$, a vortex generated by rolling-up from the pressure side to the suction side at the trailing edge causes a sharp pressure drop near the trailing edge, which is shown in Fig. 5a. After the vortex sheds from the trailing edge, the pressure at the
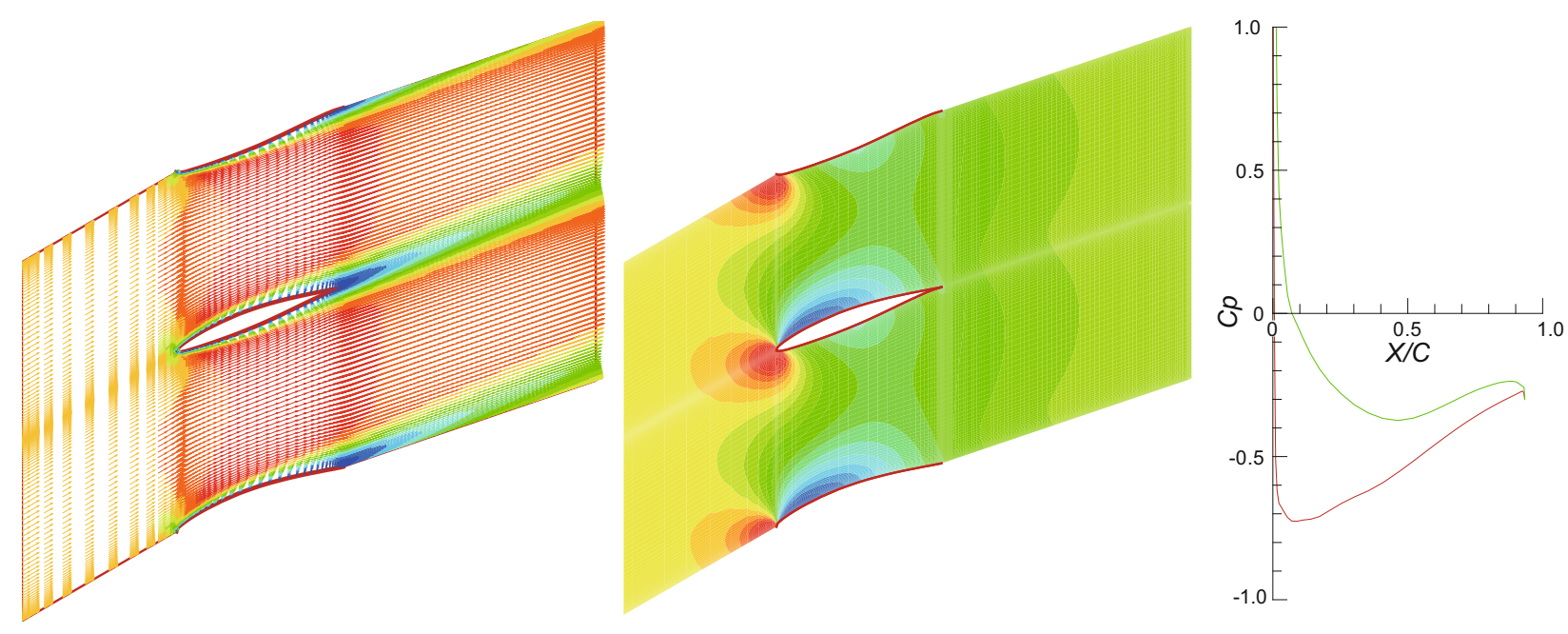

Fig. 3 Steady flow at $\alpha=9^{\circ}$ and $R e=10^{3}$
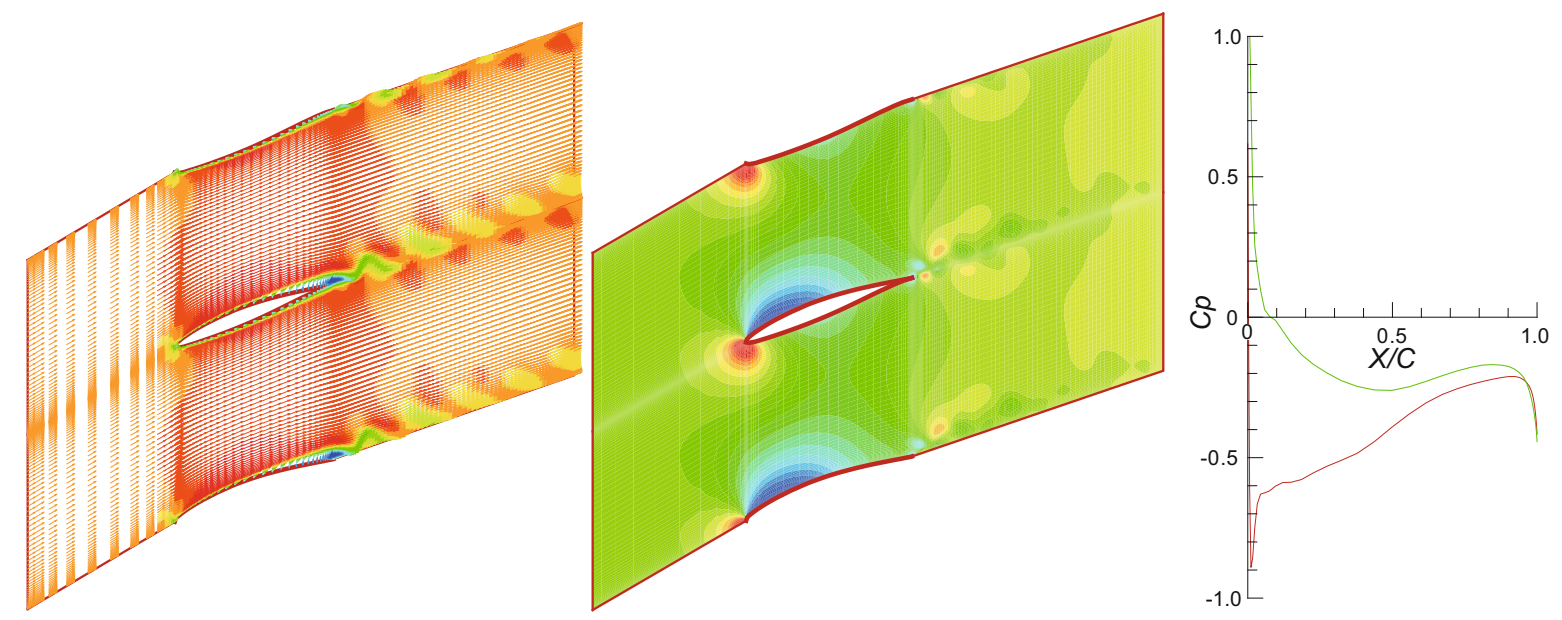

Fig. 4 Instantaneous cascade flow at $\alpha=9^{\circ}$ and $R e=10^{4}$ 
rear part of the blade tends to increase, which is shown in Fig. 5b and Fig. 5c. During this period, another vortex grows from the separated shear layer on the suction surface, which is shown in Fig. 5d and Fig. 5e. After that, a new rolled-up vortex induced by this separation vortex appears, which is shown in Fig. 5f. As a result, periodic fluctuating flow similar to the Kármán Vortex Street is formed in the wake. The period is about 0.5 .

To validate the result, in Fig. 6, the time-averaged surface pressure distribution at the angle of attack $\alpha=9^{\circ}$ and $R e=2.45 \times 10^{5}$ derived from simulation and experiment are compared. The result simulated by using the fine grid 255 $\times 75$ is more accurate than the result simulated by using the coarse grid $151 \times 75$ with respect to the experimental data. However, at the head and the trail surface of the blade, the numerical results derived from both coarse grid and fine grid differ somewhat obviously from the experimental data. A possible reason is that the $\mathrm{H}$-shaped grid is not a good fit to the blade. To investigate the unsteady flow characteriatics of the cascade, the flow fields at different angles of attack were also computed. The results are not included in this paper as space is limited.

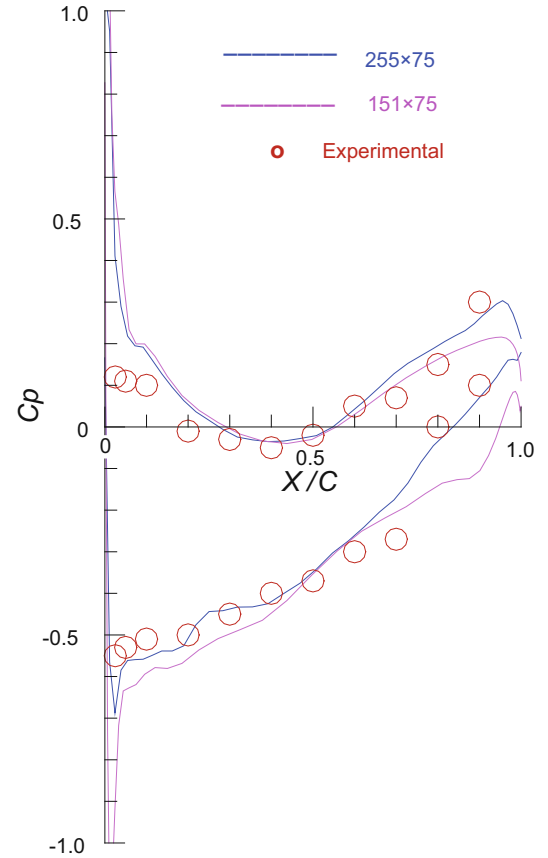

Fig. 6 Time-averaged surface pressure distribution at $\alpha=9^{\circ}$ and $R e=2.45 \times 10^{3}$

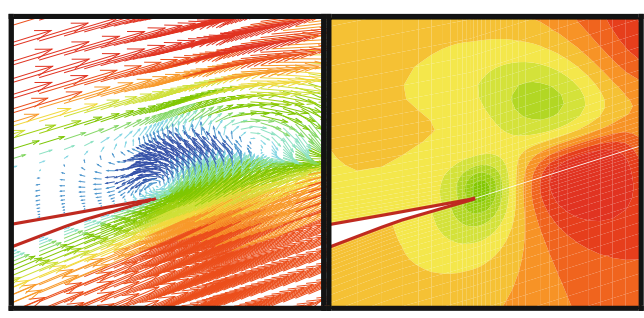

(a)

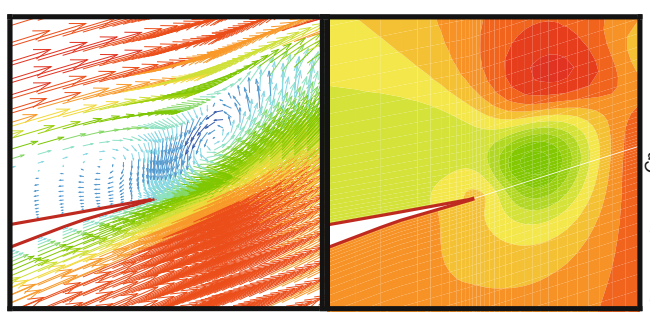

(c)

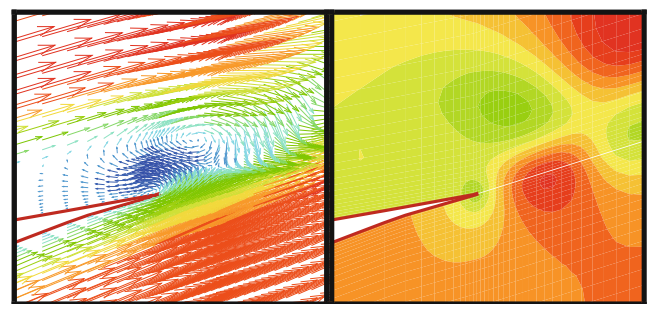

(e)

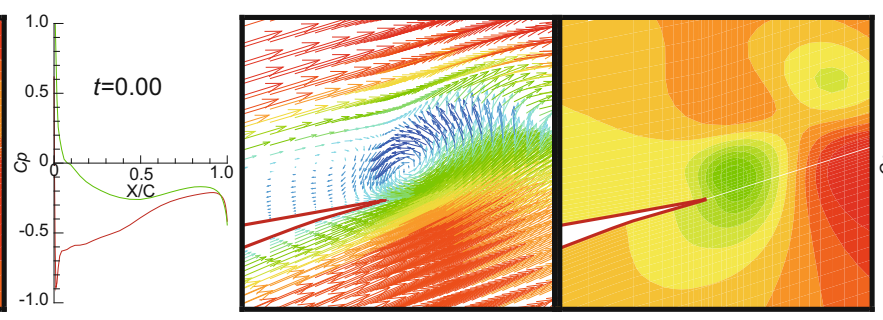

(b)
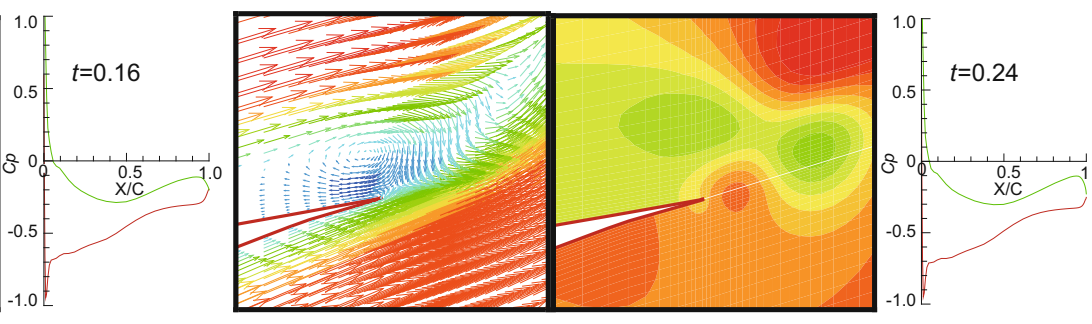

(d)
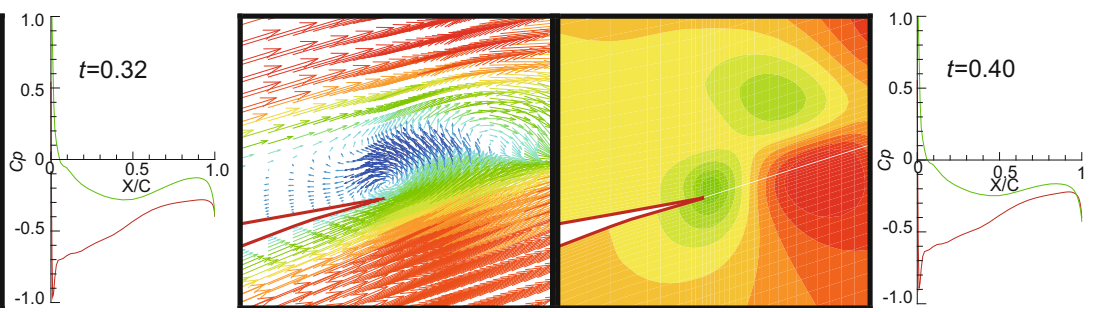

(f)

Fig. 5 Unsteady cascade flow at $\alpha=9^{\circ}$ and $R e=1.0 \times 10^{3}$ 


\section{Conclusions}

1) The present implicit SMAC scheme is applicable to simulate unsteady incompressible turbulent flow.

2) The unsteady flow of the cascade is periodic. There is flow separation on the suction side of the blade under the positive angle of attack. The Kármán Vortex Street is formed in the downstream of the cascade.

3) Reynolds number and angle of attack are two primary factors which affect the characteristics of unsteady cascade flow.

4) The computational results of surface pressure distribution are in good agreement with experimental data. The quality of the grid is one of the important factors for the accuracy of numerical simulation.

\section{References}

Amsden A A and Harlow F H. A simplified MAC technique for incompressible fluid flow calculations. J. Comp. Phys. 1970. 6(2): 322-325

Emery J C, Herrig L J, Erwin J R, et al. Systematic two-dimensional cascade tests of NACA 65-series compressor blades at low speeds. NACA Rept. 1958. No. 1368, 713-797.

Ghia K N, Osswald G A and Ghia U. Analysis of incompressible massively separated viscous flow using unsteady Navier-Stokes equations. Int. J. Numer. Methods Fluids. 1989. 9(8):1025-1050

Harlow F H and Welch J E. Numerical calculation of time dependent viscous incompressible flow of fluid with free surface. Phys. Fluids. 1965. 8(12): 2182-2189

Hou Q F and Zou S. Comparison between standard and renormalization group $k-\varepsilon$ models in numerical simulation of swirling flow tundish. ISIJ Int. 2005. 45(3): 325-330
Ismail I A and Allan M M. A designed ADI software for solving Poisson's equation. Math Comput Appl. 2004. 9(2): 167-164

Li J, Peterson G P and Cheng P. Three-dimensional analysis of heat transfer in a micro-heat sink with single phase flow. Int. J. Heat Mass transf. 2004. 47(19-20): 4215-4231

Rao P M, Kuwahara K and Tsuboi K. Computational study of unsteady viscous flow around a transversely and longitudinally oscillating circular cylinder in a uniform flow at high Reynolds numbers. Comput. Mech. 1992. 10(6): 414-428

Rogers S E. Comparison of implicit schemes for the incompressible Navier-Stokes equations. AIAA J. 1995. 33(11): 2066-2072

Rosenfeld M, Kwak D and Vinokur M. A fractional step solution method for the unsteady incompressible Navier-Stokes equations in generalized coordinates systems. J. Comput. Phys. 1991. 94(1): 102-113

Woznicki Z I and Jedrzejec H A. A new class of modified line-SOR algorithms. Journal of Computational and Applied Mathematics. 2001. 131(1-2): 89-142

Yonamine M, Ushijima T, Miyazato Y, et al. Propagation of weak pressure waves against two parallel subsonic streams. Journal of Thermal Science. 2006. 15(2): 135-139

Zhang Y X. Implicit numerical schemes and their applications based on SMAC method for computing incompressible flows. PhD Thesis. 2005a. Beijing: Tsinghua University.

Zhang Y X, Cao S L and Zhu B S. Implicit SMAC scheme for incompressible three-dimensional turbulent flow. Journal of Tsinghua University (Sci. \& Tech.). 2005b. 45(11): 1561-1564 (in Chinese)

ZhangY X, Li Z L and Zhao H B. Implicit SMAC method for unsteady incompressible turbulent flow. Journal of Mechanical Engineering. 2006. 42(11): 54-59 (in Chinese)

Zhang Y X, Zhao Y J, Cao S L, et al. Implicit SMAC scheme for the incompressible Navier-Stokes equations. Journal of Tsinghua University (Sci. \& Tech.). 2004. 44(2): 270-273 (in Chinese)

(Edited by Sun Yanhua) 